The researchers obtained the highest slope efficiency $(0.11 \%)$ in a $5.5-\mathrm{cm}$-long resonator, with an output power of $9.5 \mathrm{~mW}$ measured at $19 \mu \mathrm{W}$ launched pump power, and a minimum threshold pump power of $6.4 \mathrm{~mW}$. The researchers said that by adjusting the output coupler length, it would be possible to develop a tunable laser source. Still the researchers proposed several design enhancements, such as the addition of $\mathrm{Yb}^{3+}$ as a co-dopant to optimize pump absorption, the introduction of a loop mirror or a Bragg reflector element to emit lasing power only in one direction, and the addition of distributed feedback elements to promote singlefrequency operation. According to the researchers this would lead to smaller devices with improved efficiency, higher output power, and wavelength tunability.

JOAN J. CARVAJAL

\section{Biomimetic Nanoelectrode Demonstrates Overvoltage and Turnover Rates Compatible with PEM Technology}

The practicality of transitioning to solar and wind power relies to a large extent on efficient, reversible means of energy storage. One promising energy-storage framework is the interconversion of water and $\mathrm{H}_{2}$ in a regenerative fuel cell, where energy is stored as $\mathrm{H}_{2}$ generated by the electrolysis of water, and energy is released by the reverse reaction. Economic viability requires the development of catalysts much less expensive than $\mathrm{Pt}$, the current state of the art. Hydrogenases-naturally occurring metalloenzymes that catalyze the reversible formation of $\mathrm{H}_{2}$ from two protons and two electrons-have been investigated as alternatives to $\mathrm{Pt}$, but disadvantages, such as $\mathrm{O}_{2}$ sensitivity, are difficult to overcome. Recently, however, A. Le Goff and S. Palacin of the Commissariat à l'Energie Atomique (CEA), V. Artero of the Université Joseph Fourier of Grenoble, M. Fontecave of Collège de France, and their colleagues from various institutes and laboratories from CEA, have shown that a mimic of the active site in [NiFe]-hydrogenase attached to multiwalled carbon nanotubes (MWCNTs) results in a cathode material with a large surface area and high catalytic activity under the strongly acidic conditions typically required in proton exchange membranes (PEM).

In an article published in the December 4, 2009 issue of Science (DOI: 10.1126/ science.1179773; p. 1384), Artero and coresearchers cited the advantages of MWCNTs, which include large surface areas that facilitate high catalyst loading, stability, and electrical conductivity, as well as simple methods for binding complexes onto their surfaces. The researchers prepared electrodes by first depositing a thin film of MWCNTs onto an indium tin oxide substrate using a previously published soluble membrane technique. Scanning electron microscopy was used to demonstrate a large surface area composed of MWCNT bundles with extensive branching. Electro-reduction of a diazonium salt was used to covalently attach

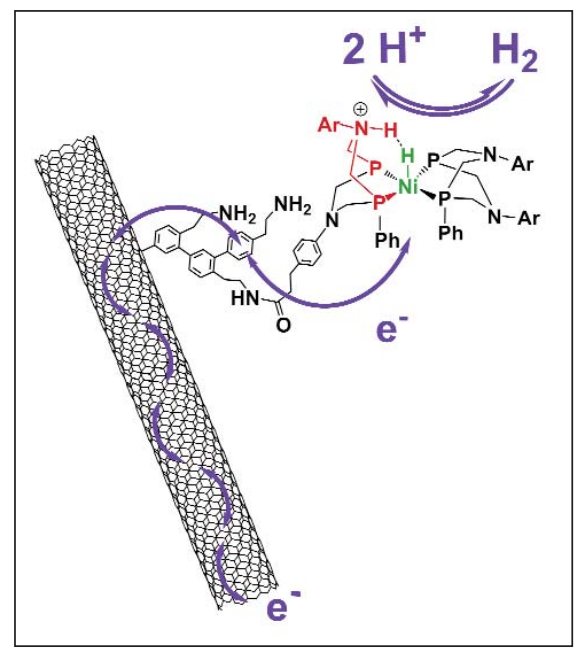

Figure 1. A schematic representation of the structure of the bio-inspired, $\mathrm{H}_{2}$ evolving nickel catalyst grafted onto a multiwalled carbon nanotube (MWCNT). Activated ester moieties have been introduced in the Aryl (Ar) groups to enable the covalent coupling of the catalyst through amidation with the aminedecorated MWCNTs. Blue arrows trace the electron transport chain toward the active site through the conductive MWCNT. The ligands acting as proton relays in heterolytic $\mathrm{H}_{2}$ evolution reaction are depicted in red, while the metal center stabilizing hydride ions are depicted in green. The number of phenylene residues shown is arbitrary and the researchers do not exclude attachment of the nickel complex to two or more surface amine groups. From Science 326 (December 4, 2009) 1384; DOI: $10.1126 /$ science. 1179773.

Reprinted with permission from AAAS. (C) 2009 AAAS.

4-(2-aminoethyl)phenyl groups onto the surface of the electrodes. The presence of amine and ammonium groups was verified with x-ray photoelectron spectroscopy (XPS). The researchers then anchored onto the amino-functionalized MWCNT electrode a [Ni-Fe]-hydrogenase-inspired nickel catalyst (see Figure 1), initially developed by D.L. DuBois (Pacific Northwest National Laboratory) and shown to be stable in air and to moisture. XPS analysis confirmed the presence of the intact $\mathrm{Ni}$ complex at the electrode surface. Cyclic voltammetry displayed a cathodic process that the researchers assigned to the reduction of the $\mathrm{Ni}(\mathrm{II})$ complex.

Controlled-potential coulometry in nonaqueous media was used to demonstrate the molecular electrode material's stability and its ability to sustain catalysis of $\mathrm{H}_{2}$ production. At a potential of $-0.5 \mathrm{~V}$ versus normal hydrogen electrode, an electrochemical cell produced $\mathrm{H}_{2}$ with a sustained current density of $2 \mathrm{~mA} / \mathrm{cm}^{2}$, producing 15 mmoles of $\mathrm{H}_{2}$ in 1 hour, which corresponds to a turnover rate of approximately 20,000.

The researchers also demonstrated that the molecular electrode material can be used in diluted sulfuric acid (with a $\mathrm{pH}$ in the range of 0 to 1 ) using a membraneelectrode assembly similar to that developed for proton-exchange membrane electrolyzers but with the $\mathrm{Pt} / \mathrm{C}$ catalyst replaced by Ni-functionalized MWCNTs. In that case the electrode support is a gas diffusion layer made of carbon fibers and after deposition of the catalytic material, the electrode is covered with a Nafion membrane to protect it from the solution while allowing protons to access the catalyst. Hydrogen evolves from this electrode with a very low overvoltage (i.e., almost at thermodynamic equilibrium) which is key for a good energetic efficiency of the device.

The researchers observed a constant current density throughout a 10-hour experiment with more than 100,000 turnovers. The researchers also showed that, under these conditions, their $\mathrm{Ni}$ functionalized MWCNTs can catalyze the reverse reaction, that is, $\mathrm{H}_{2}$ oxidation.

The researchers said, "Our results show that the biomimetic nanomaterial reported here already rivals with platinum as far as overvoltage is concerned and offers promising turnover rates under conditions compatible with widespread PEM technology on the basis of commercial proton exchange membranes in acidic electrolytes."

STEVEN TROHALAKI 